\title{
Internal kinematics and binarity of X-ray stars in the Pleiades open cluster
}

\author{
V. V. Makarov ${ }^{1}$ and N. Robichon ${ }^{2}$ \\ 1 Copenhagen University Observatory, Juliane Maries Vej 30, 2100 Copenhagen $\varnothing$, Denmark \\ 2 DASGAL, Observatoire de Paris-Meudon, bât. 11, 5 place Jansen, 92195 Meudon Cedex, France
}

Received 27 November 2000 / Accepted 10 January 2001

\begin{abstract}
The classical convergent point analysis is implemented for the Pleiades stars with proper motions in the Tycho-2 Catalogue and X-ray fluxes measured by the ROSAT satellite. It is demonstrated that, with the standard astrometric errors as given in Tycho-2, strong X-ray sources in the cluster $\left(\log L_{\mathrm{X}}>29.1\right.$, where $L_{\mathrm{X}}$ is in erg s $\mathrm{s}^{-1}$ ) exhibit a velocity dispersion in one component of only $0.20 \mathrm{~km} \mathrm{~s}^{-1}$, while the distributions of velocity components of moderate $\left(\log L_{\mathrm{X}}<29.1\right)$ sources and stars not detected by ROSAT at all are consistent with a velocity dispersion of $0.64 \mathrm{~km} \mathrm{~s}^{-1}$. The difference is statistically significant at the level of $1.6 \sigma$, or 0.95 confidence limit. This result is a clue to the kinematics/X-ray luminosity segregation, similar to that previously discovered in the Hyades open cluster. It is discussed that the segregation may be caused by a wide spread of ages of the member stars. The occurrence of high X-ray luminosities is found to correlate very well with visual binarity and multiplicity (separations $>10 \mathrm{AU})$.
\end{abstract}

Key words. open clusters and associations: individual: Pleiades - stars: kinematics - astrometry

\section{Introduction}

The Pleiades is a key stellar formation for many Galactic studies. The cluster is one of the youngest in the Solar neighbourhood (age $\sim 100 \mathrm{Myr}$ ) and is conveniently close to the Sun, allowing rather detailed study. The Hyades cluster is optimal an internal kinematics investigation, and quite accurate estimations of the intrinsic velocity dispersion have been obtained (Perryman et al. 1998; Makarov et al. 2000), in reasonable agreement with the theoretically predicted $0.23 \mathrm{~km} \mathrm{~s}^{-1}$ (Gunn et al. 1988) for a state of dynamical equilibrium. For the Pleiades, Jones (1970) determined a somewhat higher dispersion, $\sigma_{v}=$ $0.42 \mathrm{~km} \mathrm{~s}^{-1}$, in one velocity component. He investigated the motions of stars of various masses in the cluster and found no increase in $\sigma_{v}$ for stars of smaller masses. His conclusion was that "it is unlikely that there is equipartition of kinetic energy among the stars of various masses". From the value of $\sigma_{v}$ and the Virial theorem he derived a total mass of $690 M_{\odot}$, while the direct counting of stars resulted in $460 M_{\odot}$. A more recent determination by Geffert et al. (1995) resulted in a substantially higher estimate at $1 \mathrm{mas} / \mathrm{yr}=0.6 \mathrm{~km} \mathrm{~s}^{-1}$. This is rather on the high side of what would be expected of a cluster in dynamical equilibrium. However the assumption of dynamical equilibrium

Send offprint requests to: V. V. Makarov,

e-mail: makarov@astro.ku.dk should not be taken for granted in a young cluster like the Pleiades.

Our present analysis is based on the astrometric data in the Tycho-2 catalogue (Høg et al. 2000a). The catalogue includes 2.5 million stars distributed over the entire sky. Proper motions are derived for 96 percent of the stars from the observed positions in Tycho-2, the Astrographic Catalogue and 143 other ground-based catalogues (Høg et al. 2000b). The system of Tycho-2 proper motions is tied to that of Hipparcos systematically, on the large and intermediate scales. As far as accidental errors for individual stars are concerned, the Tycho-2 and Hipparcos proper motions are statistically independent, i.e. uncorrelated.

Even though the positional precision of Tycho-2 observations and other source catalogues is poorer, the duration of the observation record ( $\sim 100$ years) makes the Tycho- 2 proper motions even more precise than those of Hipparcos (ESA, 1997), for some stars. The median formal standard errors of Tycho-2 proper motions are equal to $1.0 \mathrm{mas} / \mathrm{yr}$ for stars with Tycho $V_{\mathrm{T}}$ magnitude brighter than 7 , and $1.5 \mathrm{mas} / \mathrm{yr}$ for stars with $V_{\mathrm{T}}$ between 9 and $10 \mathrm{mag}$. An important asset of Tycho-2 proper motions in the context of this work is that they are less prone to the perturbations from orbital motion in binaries, as was shown in Makarov et al. (2000) using the example of the Hyades. A Tycho-2 proper motion is rarely perturbed by an orbital motion 
with a period $\ll 100$ yr. In the Pleiades, spectroscopic binaries will not hamper our kinematic analysis, for their periods are shorter than 1000 d (Mermilliod et al. 1992), but they may certainly be a threat for the Hipparcos data based on 3.5 yr of observation.

We cull our sample of stars from the two ROSAT surveys of the Pleiades (Stauffer et al. 1994; Micela et al. 1999) by direct positional cross-identification with the Tycho- 2 catalogue. The survey by Stauffer et al. is based on three deep images within the cluster obtained with the PSPC (Position Sensitive Proportional Counter) instrument. They detected $317 \mathrm{X}$-ray sources, 171 of which were identified with cluster members. The work by Micela et al. dwells on deep images of the cluster obtained with the HRI (High Resolution Imager). Although positionally the HRI images overlap with the larger PSPC fields, more X-ray stars were identified due to greater sensitivity. Whenever a Tycho star was found in both surveys, preference was given to the Micela et al. data. Three stars, TYC 1247-0076-1, 1260-0671-1 and 1813-0126-1, all of which are strong X-ray sources situated outside the PSPC and HRI images, were found in the ROSAT AllSky Survey Bright Source Catalogue (Voges et al. 1999) and were included in the sample. Finally, only those stars that contain proper motions in the Tycho- 2 catalogue were used, bringing the total number in the sample to 87 .

The Tycho-2 catalogue is statistically complete with stars brighter than $V=11.5 \mathrm{mag}$. We discovered, however, that the degree of completeness varies strongly with position on the sky. For example, when stars from the Supplement-1 to the catalogue are plotted, which contains bona fide stars from Hipparcos and Tycho-1 (Høg et al. 1997) missing in Tycho-2, very prominent concentrations of number density are seen at the location of rich open clusters. It appears that a major fraction of stars brighter than $V=11.5$ missing in Tycho-2 are associated with such open clusters. For the Pleiades, up to $25 \%$ of sufficiently bright members of the cluster in common with the X-ray surveys are missing in the main Tycho-2 catalogue. A few stars are missing altogether; 20 stars are given in the Supplement- 1 to the catalogue, and 2 stars are in the main catalogue but without proper motions. The stars in the Supplement-1 have proper motions in the ACT reference catalogue (Urban et al. 1998). The reason for these missing stars in Tycho- 2 was that the authors of the catalogue applied very strict criteria in accepting astrometric solutions (Høg et al. 2000c). If a solution appeared to be much disturbed by interfering nearby stars, especially at some specific distances from bright companions where side lobes and ghost images from other astrometric slits might occur, it was discarded. Obviously, in a dense cluster like the Pleiades, populated by quite bright stars, this stringency led to rejection of many genuine stars. Although for most of them, proper motions of comparable precision could be found in the ACT, we decided against their inclusion in the sample. The ACT proper motions have been proven inferior to those in Tycho- 2 in the systematic respect due to a different way of treating the Astrographic
Catalogue data (Urban et al. 2000). In order to detect the very subtle differences in the internal kinematics of the cluster, astrometric data as uniform and accurate as possible are required.

\section{Internal velocity dispersion}

We are using a variation of the classical moving cluster method (e.g., Smart 1938), based on the assumption of a common motion of cluster stars in the same direction and with the same velocity. The reader can find a more detailed description of this method in Makarov et al. (2000). We note in passing that this particular variation of the classical method has the advantage of exploiting the proper motions of cluster stars to the largest possible extent. Proper motions from the Tycho- 2 catalogue are the most accurate data available at present regarding internal cluster kinematics studies. Radial velocities and trigonometric parallaxes are less accurate, and they are used here only for scaling distances and velocities in physical units.

For a given star in the sample, its coordinates $(\alpha, \delta)$ and proper motion components $\left(\mu_{\alpha *}, \mu_{\delta}\right)$ are known, in the equatorial coordinate system, where $\mu_{\alpha *}$ denotes $\mu_{\alpha} \cos \delta$. The proper motion components define a proper motion vector $\boldsymbol{\mu}$ tangential to the celestial sphere at the given point. When many stars' vectors utilized and extended around the celestial sphere, they meet at and around the common convergent point of the cluster, which defines the direction of the centroid motion with respect to the Sun. The first step of our procedure is determining the coordinates of the convergent point by a purely geometrical method. For each star, the differences, $\Delta$, between the tangential direction of the proper motion vector and the direction from the star position to the convergent point is computed. The expected dispersion of $\Delta$ is

$\sigma_{\Delta}^{2}=\frac{\sigma_{\mu \perp}^{2}}{\mu^{2}}+\frac{\sigma_{v}^{2}}{v^{2} \sin ^{2} \lambda}$,

where $\sigma_{\mu \perp}^{2}$ is the standard error of the proper motion component perpendicular to the convergent point direction for the given star, $\mu=|\boldsymbol{\mu}|, \sigma_{v}$ is the internal velocity dispersion in one component for this cluster, assumed isotropic, $v$ is the magnitude of the centroid velocity, and $\lambda$ is the angular distance between the star and the convergent point. In determining the convergent point coordinates, we find a point which provides the minimum quadratic sum of the normalized differences $\Delta / \sigma_{\Delta}$, assuming a certain $\sigma_{v}$ (null for the Pleiades sample) and iteratively rejecting deviant stars beyond $2.5 \sigma_{\Delta}$. This is essentially a $\chi^{2}$ fitting. In cases of strongly non-Gaussian distributions, smaller norm solutions could give a more robust result, e.g., 1norm or 1.5-norm solutions. These solutions were tried for the given sample, but they produced similar results. In order to determine the convergent point to the maximal accuracy, we add to the sample of 87 stars with measured X-ray fluxes, 34 Hipparcos stars from the list of members in Robichon et al. (1999), which are outside 

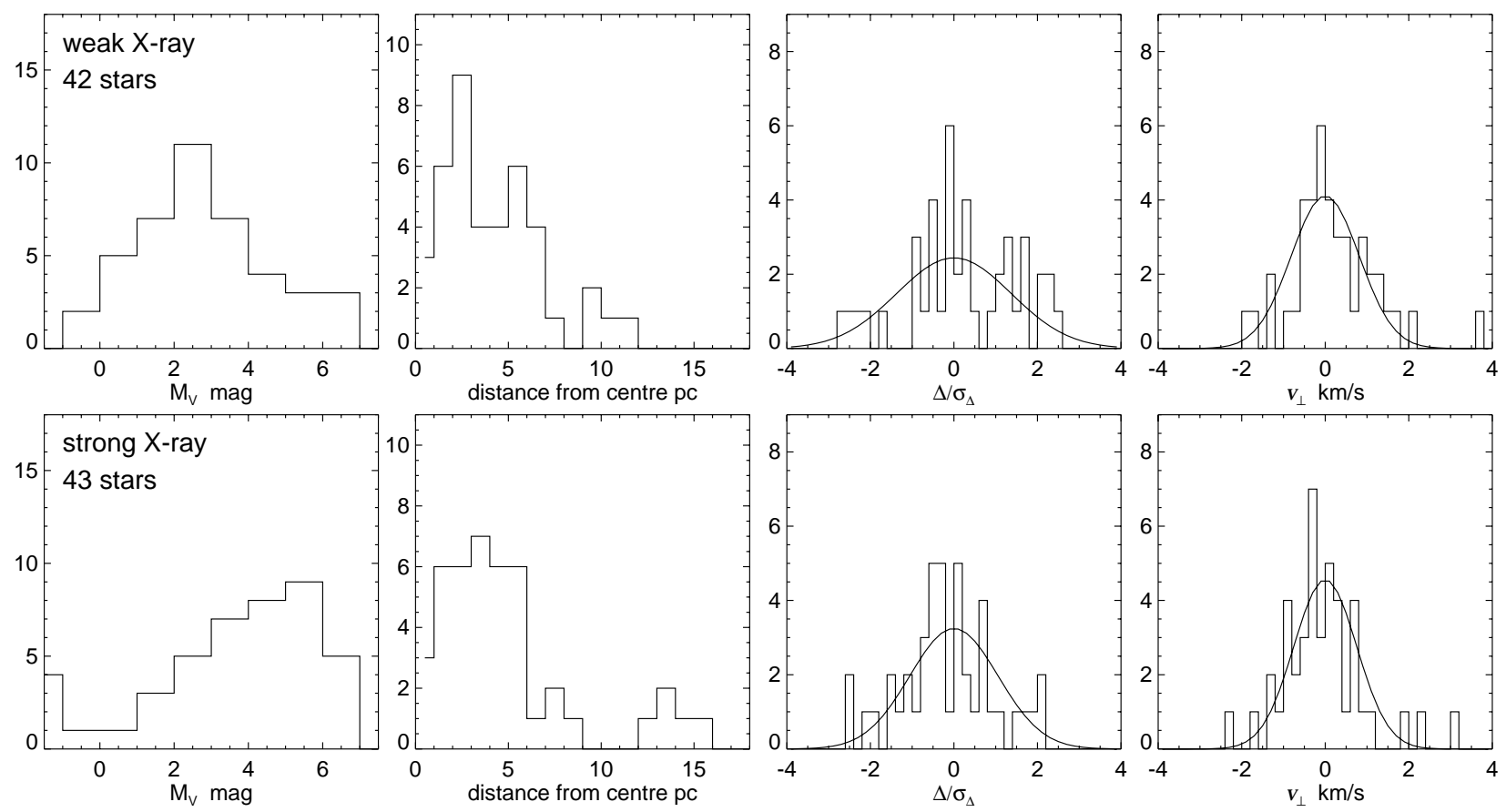

Fig. 1. Photometric and kinematic histograms for the Pleiades stars with proper motions in the Tycho-2 catalogue. The following distributions are shown in columns, left to right: 1) absolute $V$ magnitudes as derived from visual magnitudes in the literature and 'kinematic' distances from the moving cluster method; 2) distances from the cluster centre; 3) relative deviations of the proper motion direction from the convergent point direction, $\left.\Delta / \sigma_{\Delta} ; 4\right)$ observed transverse velocity component $v_{\perp}$ in $\mathrm{km} \mathrm{s}^{-1}$. Full lines in the last two columns show least-squares Gaussian fits to the observed histograms, assuming for $\Delta / \sigma_{\Delta}$ a zero velocity dispersion. The two rows of graphs correspond to the non-overlapping subsets 1) stars not detected in X-rays and detected with X-ray luminosities below $1.2610^{29} \mathrm{erg} \mathrm{s}^{-1} ; 2$ ) stars detected with X-ray luminosities above $1.2610^{29} \mathrm{erg} \mathrm{s}^{-1}$. Two stars with $\Delta / \sigma_{\Delta}>3.5$, both in the first subset, were deleted from the analysis

the ROSAT images. This provides a much larger angular extent of the sample across the sky and gives a safer determination in the direction from the cluster centre to the convergent point. With the extended sample of 121 stars, the $\chi^{2}$ solution gives $\left(\alpha_{\mathrm{CP}}, \delta_{\mathrm{CP}}\right)=\left(92^{\circ} .49,-47^{\circ} .87\right)$ with formal errors $(5.4,5.3)$, but in fact the ellipse of covariance is strongly elongated along the line connecting the centre of the cluster and the convergent point. The largest component standard error is 6.4 along this line. The average $\lambda$ is 78.8 .

The distance $D$ to each star in parsec is obtained from the relation

$v \sin \lambda=4.74 \mu D$,

where $\mu$ is in $\operatorname{arcsec} \mathrm{yr}^{-1}$, and $v$ is the centroid velocity of the cluster. Conversely, the centroid velocity can be derived from stars with known trigonometric or other parallaxes. The alternative is to use measured radial velocities related to $v$ by $v_{\mathrm{R}}=v \cos \lambda$. It is natural to use the Hipparcos parallaxes of the Pleiades for the calibration of $v$. The distance to the Pleiades became the subject of a controversy since 1997 when the Hipparcos catalogue was published. The Hipparcos mean distance of $118 \pm 3 \mathrm{pc}$, derived from the mean parallax of 54 stars (Robichon et al. 1999), is in disagreement with photometric determinations by the main sequence fitting method (Loktin \& Matkin 1994; Pinsonneault et al. 1998) and ground-based parallax measurements (Gatewood et al. 2000). Most of the nonHipparcos determinations agree on the distance $135 \pm 5 \mathrm{pc}$, with the difference from the Hipparcos mean being 5 times the formal error of the latter. Important issue as it is, the true distance of the cluster has limited bearing on the results of this work. If the true distance is greater by 10 to $15 \mathrm{pc}$, the physical estimates should be simply scaled accordingly, e.g., the centroid velocity, velocity dispersions, distances from the cluster's centre should be increased by the same factor, and X-ray luminosities by its square. The basic conclusions regarding the kinematic segregation of X-ray stars would not be changed.

The CORAVEL mean radial velocity of $+5.7 \pm$ $0.5 \mathrm{~km} \mathrm{~s}^{-1}$ and the equatorial coordinates of the convergent point at $\left(92^{\circ} 49,-47^{\circ} .87\right)$, as determined strictly from the geometry of the proper motion vectors, imply a centroid velocity of about $30 \mathrm{~km} \mathrm{~s}^{-1}$. The average distance to the cluster is then computed to be $126 \mathrm{pc}$, right in between the Hipparcos mean distance of 118 pc and the pre-Hipparcos estimates. This estimate is statistically very poor, the standard errors being $(-47,+171)$ pc from the uncertainty of $\lambda$ alone. Although we stick to this mean 
distance in the following, it is concluded that the convergent point method does not permit us to put useful constraints on the true distance to the Pleiades due to the smallness of the radial velocity component.

The centroid velocity is $\boldsymbol{v}=(-0.8,+19.1$, -20.9) $\mathrm{kms}^{-1}$ with respect to the Sun. In the Galactic coordinates, the velocity ${ }^{1}$ is $(U, V, W)=$ $(-6.7,-26.0,-13.4) \mathrm{km} \mathrm{s}^{-1}$, very close to Robichon et al.'s estimation. With these mean parameters of the cluster, Eq. (2) can be used to estimate the kinematic distance $D$ for each star. Formally, its relative precision is much higher than that of the Hipparcos individual parallaxes, because the relative error of the proper motions is only about $2 \%$, compared with the $\sim 15 \%$ relative error of the parallaxes.

For the following kinematic analysis, we estimate the transverse velocity component $v_{\perp}$ for each star, i.e. the tangential velocity components normal to the convergent point direction,

$v_{\perp}=v \sin \lambda \sin \Delta$.

We select 85 Tycho- 2 stars out of the original 87 with $\Delta / \sigma_{\Delta}$ less than 3.5, computed under the basic assumption of a $\sigma_{v}=0$. Both rejected stars, TYC 1800-1675-1 $=$ HII 1675 and TYC 1800-1567-1 = HII 1397, are nondetections in the ROSAT data ${ }^{2}$. The 87 stars are divided into two roughly equal partitions: 1) 44 stars not detected in X-rays and those detected with $\log L_{\mathrm{X}}$ less than 29.1; 2) 43 stars with $\log L_{\mathrm{X}}>29.1$ ( $L_{\mathrm{X}}$ is always expressed in $\operatorname{erg~s}^{-1}$ and corrected for the kinematic distance in this paper). The distributions of absolute $V$ magnitudes, distances from the cluster centre, relative angular deviations from the convergent point direction and transverse velocity components in the two partitions are shown in Fig. 1. The position vector of the cluster centre is assumed to be $(63.0,95.8,51.6) \mathrm{pc}$ in the ICRS. Not much difference is seen in the distributions of stars relative to the centre (second column of plots), but the reader should note that the X-ray surveys in use cover only the central part of the cluster in the celestial projection. The distributions of $v_{\perp}$ set absolute upper bounds on the internal dispersion. The Gaussian least-squares fits have standard deviations of 0.82 and $0.75 \mathrm{~km} \mathrm{~s}^{-1}$ for the two partitions. The physical dispersion of velocities is significantly smaller, because the astrometric measurement error is the dominating component of the total observed dispersion.

Assuming a certain $\sigma_{v}$, the distribution of the $\Delta / \sigma_{\Delta}$ values can be computed from Eq. (1). If the assumption is correct and the formal errors are realistic, the standard deviation of this distribution should be close to unity. The upper row in Fig. 1 shows such distributions and their least squares fits with a $\sigma_{v}=0$. The estimated standard deviations are 1.37 and 1.06. The width of the distributions for the two partitions can be brought down to 1 with

\footnotetext{
1 The $U$ axis is pointing towards the Galactic centre.

2 HII numbers are conventional identifications from the catalogue by Hertzsprung (1947).
}

$\sigma_{v}=0.20$ and $0.64 \mathrm{~km} \mathrm{~s}^{-1}$, respectively. Thus, our data tell us that the strong X-ray sources are much less dispersed in their internal velocities than the weak sources.

Is this result statistically reliable? Assuming that $\Delta / \sigma_{\Delta}$ is a sample of a normal population, a standardized normal statistics approximation may be used to set confidence intervals of the variance estimates (Kendall \& Stuart 1973, vol. 2, Example 20.6). For example, it is found that the probability of the standard deviation over the first sample (weak X-ray) being less than 1.22 is 0.12 , the same as the probability of the standard deviation of the second sample (strong X-ray) being greater than 1.22 . The 0.67 confidence interval, corresponding to a $\pm 1 \sigma$ interval, is $[1.24,1.55]$ for the first sample standard deviation, and $[0.96,1.20]$ for the second. The combined error of the difference between the estimated standard deviations $(1.37-1.06=0.31)$ is, roughly, 0.19 , which corresponds to a $1.6 \sigma$, or $95 \%$ confidence, level. Alternatively, the approximated confidence intervals may be used to compute the probability that our basic conclusion is wrong, i.e., that the variance over the first sample of $\Delta / \sigma_{\Delta}$ is in fact smaller than the second one. This probability is only 0.04 .

A completely different way to estimate the dispersion is the percentile method, that is to scale the distribution so that $32 \%$ of the sample is outside the $\pm 1 \sigma$ interval, as expected for a normal distribution. The percentile method is known to produce conservative (overestimated) values for non-Gaussian distributions. However, the Gaussian fit estimates are confirmed by percentiles, since only 14 stars out of 43 deviate beyond $1 \sigma$ in the first partition, and 16 out of 44 in the second, when their estimated $\sigma_{v}$ are assumed.

\section{X-ray status of the binaries}

For the present analysis of binaries we make a finer division of the 87 stars into 3 groups:

\section{34 non-detections in X-rays;}

2. 25 detected sources with $\log L_{\mathrm{X}}<29.4$;

3. 28 detected sources with $\log L_{\mathrm{X}}>29.4$.

There are 34 stars in our sample of 80 in common with the lists of members in Mermilliod et al. (1992) and Raboud \& Mermilliod (1998), spectroscopically observed with CORAVEL. Twenty of these stars have no trace of duplicity in the CORAVEL data, 10 are spectroscopic binaries with periods shorter than $1000 \mathrm{~d}, 2$ stars are suspected binaries due to photometry but not confirmed spectroscopically, 1 star (TYC 1804-1179-1 = HII 2278) is detected as binary by infra-red imaging (Bouvier et al. 1997), and 1 star (TYC 1800-1846-1 = HII 1912) is a visual binary. Counting the two photometrically suspected stars as singles, the proportion of singles to doubles is $22: 12$, i.e. virtually the same as the 56:30 determined by Mermilliod et al. (1992).

However, the proportion of binaries varies significantly among the 3 groupings by X-ray luminosity. It is quite high 
Table 1. Visual binaries in the sample of X-ray selected stars in the Pleiades with proper motions in Tycho-2. The information on binarity is culled mainly from Mason (1993) and Turon et al. (1992). One star, TYC 1803-1061-1, is a new discovery in this paper. Identification numbers in the Tycho-2, (Hertzsprung 1947) and Hipparcos catalogues are given in Cols. 1 through 3. Spectral types from the Simbad data base are given in Col. 4. A mean distance to the cluster of $118 \mathrm{pc}$ is assumed while calculating $\log L_{\mathrm{X}}$ in Col. 5. Relative deviations of proper motion vectors from the direction to the convergent point, $\Delta / \sigma_{\Delta}$, are in Col. 6. Separations between the components in AU in Col. 7 were computed assuming the kinematic distance for each star

\begin{tabular}{|c|c|c|c|c|c|c|}
\hline TYC & HII & HIP & Sp. type & $\log L_{\mathrm{X}}$ & $\Delta / \sigma_{\Delta}$ & Sep \\
\hline 1803-1061-1 & 514 & & $?$ & 29.25 & +0.63 & $\begin{array}{l}\text { AU } \\
100\end{array}$ \\
\hline 1803-1585-1 & 563 & 17531 & B6IV & 30.01 & -0.11 & $5 ; 9300$ \\
\hline 1799-0184-1 & 717 & & A1V & 28.98 & +1.35 & 27 \\
\hline 1799-1005-1 & 801 & 17572 & $\mathrm{~A} 0 \mathrm{~V}$ & 29.20 & -1.05 & 420 \\
\hline $1800-1961-1 \& 2$ & 956 & & $\mathrm{~A} 7 \mathrm{~V}$ & 29.89 & +0.03 & 115 \\
\hline $1800-2204-1$ & 980 & 17608 & B6IVe & 29.80 & +1.74 & $?$ \\
\hline 1800-1908-1 & 1028 & & $\mathrm{~A} 2 \mathrm{~V}$ & $<28.35$ & -2.41 & 20 \\
\hline 1804-2081-1 & 1234 & 17664 & B9.5V & 29.31 & -0.19 & 25 \\
\hline 1804-2095-1 & 1266 & & A9V & 29.64 & -2.41 & 66 \\
\hline $1800-2201-1$ & 1375 & & $\mathrm{~A} 0 \mathrm{~V}$ & $<29.25$ & -0.08 & 9800 \\
\hline $1800-1546-1$ & 1762 & & A9V & 29.68 & -0.40 & 10 \\
\hline $1800-1846-1$ & 1912 & & $\mathrm{~F} 4 \mathrm{~V}$ & 29.56 & -1.93 & 39 \\
\hline $1800-2203-1$ & 2168 & 17847 & B8III & 29.67 & +0.63 & $0.5 ; 51$ \\
\hline $1800-2200-1$ & 2181 & 17851 & B8Vpe & 29.67 & +0.00 & 27 \\
\hline 1804-2047-1 & 2263 & 17862 & B9.5V & $<28.48$ & +1.05 & 11000 \\
\hline 1804-1179-1 & 2278 & & K0 & 29.53 & -1.02 & 45 \\
\hline $1800-1260-1$ & 2500 & 17923 & $\mathrm{~A} 0 \mathrm{~V}$ & 30.05 & -0.46 & 390 \\
\hline
\end{tabular}

in the first group (non-detections), 4:4, and in the third group (strong X-ray), 5:4. The moderate X-ray stars are depleted in binaries with the proportion 13:4. It appears from this very different incidence of spectroscopic binarity that there is a certain physical difference between the moderate and strong X-ray stars. Some part of this difference may be related to the spectral type census of these groups.

To the number of spectroscopic binaries one could add the short period binary TYC 1800-2203-1 = HIP 17847 $=$ HII 2168 which has an "orbital solution" in HIP with a period of $291 \pm 9 \mathrm{~d}$ and semimajor axis $\sim 0.5 \mathrm{AU}$. It is a B8III star, remarkably bright in X-rays $\left(\log L_{\mathrm{X}}=\right.$ $29.67)$ with unperturbed proper motion $\left(\Delta / \sigma_{\Delta}=0.63\right)$. This addition corroborates our conclusion that incidence of high X-ray activity is well correlated with short-period binarity. The existence of the near component was first detected by Meyer et al. (1995) at projected separation $\sim 6$ mas. In fact, this star is likely triple, because on-ground observations also detect a visual binary with separation about 0.4 arcsec (e.g., Turon et al. 1992).

Among the 10 spectroscopic binaries in the sample, 7 have $\left|\Delta / \sigma_{\Delta}\right|<1$, assuming a zero intrinsic velocity dispersion. This fact reinforces our proposal that short pe- riod orbital motion does not perturb the Tycho-2 proper motions at all.

Relatively fewer visual double stars are known in the Pleiades than in the Hyades. They are surely harder to resolve by speckle interferometry, being 3 times more distant. The proportion of binaries at separations 5 to $50 \mathrm{AU}$ appears to be well correlated with the age of a stellar aggregate, and in the Hyades the companion star fraction is estimated at 0.30 , which is intermediate between the values for $\mathrm{T}$ Tauri stars (0.40) and nearby $\mathrm{G}$ dwarfs (0.14) (Patience et al. 1998). In the Pleiades, separations less than $\sim 10 \mathrm{AU}$ are rather out of reach for Hipparcos, requiring an angular resolution better than 80 mas.

To the couple of already-mentioned visual binaries in the present sample we add 15 more systems discovered by speckle interferometry (Mason et al. 1993) and other ground-based techniques. One star, TYC 1803-1061-1 = HII 514, is resolved for the first time by us from the raw Tycho-2 data, using the routine double star reduction software described in $\mathrm{H} \varnothing \mathrm{g}$ et al. (2000c). The detected component is found at 0.78 arcsec separation and $58^{\circ}$ position angle. The $V_{T}$ magnitudes are 11.4 and 11.6. All 17 known visual binaries and triples in our X-ray selected sample are listed in Table 1. Together with the spectroscopic binaries, they amount to 26 multiple systems (the star TYC 18001260-1 = HII 2500 is known as a wide triple, but it also seems to be a spectroscopic binary, which makes it quadruple).

Stauffer et al. (1994) found a strongly bimodal distribution of $\log L_{\mathrm{X}}$ for stars with $B-V<0.35 \mathrm{mag}$ (cf. their Figs. 12 and 13). They failed to find blue stars in the interval of $\log L_{\mathrm{X}} 28.8$ to 29.2. A similar gap, but at lower luminosities, was observed in the Hyades (Stern et al. 1995, Fig. 8). The gap is not so distinct in our 3 partitions, where 46 stars with $B-V<0.35$ are distributed as 25:9:12. A few stars with $L_{\mathrm{X}}$ measured by Micela et al. (1999) and corrected for kinematic distances fill the gap. Still, there seems to be a well-defined group of blue stars strong in X-rays. Since these stars are devoid of outer convective envelopes, they must generate X-rays in a completely different way from stars later than $G$, where the non-thermal dynamo mechanism is at work. Stauffer et al. therefore suggest that the X-ray active early-type stars have low-mass binary components, responsible for the high $L_{\mathrm{X}}$. Indeed, among the 12 stars with $B-V<0.35$ and $\log L_{\mathrm{X}}>28.45$ (third partition), the proportion of single (as presently known) to visual resolved binaries is 5:7. For comparison, the proportion for non-detections (partition 1) is $24: 2$.

Moreover, there is a striking correlation between the occurrence of high X-ray luminosities and visual binarity. If $\log L_{\mathrm{X}}$ and binarity were uncorrelated, the distribution of the visual binaries over the three partitions would be close to $34: 25: 28$. The observed distribution is $3: 3: 11$ (Table 1). We draw the conclusion, that the highest X-ray luminosities are strongly associated with visual, i.e., wider separation binarity. 
Two stars, TYC 1803-1585-1 and 1800-1260-1, have $L_{\mathrm{X}}$ markedly higher than the other $\mathrm{B}$ and early $\mathrm{A}$ stars. TYC 1800-1260-1 is quadruple, which might explain its outstanding brightness. One may suspect that the HIP 17531 has a spectroscopic component too, i.e. it is triple. On the other side of the $\log L_{\mathrm{X}}$ distribution in Table 1, three stars, HII 1028, 1375 and 2263 are not detected by ROSAT. The companion of the latter, HII 2220, is also present in our sample as a non-detection. HII 1375 forms a quadruple system with Alcyone (TYC 1800-2202-1, not present in Tycho-2), TYC 1800-1607-1 (also non-detection by ROSAT) and TYC 1800-2222-1 $\left(\log L_{\mathrm{X}}=29.40\right)$. HII 1028 is quite interesting, having $\Delta / \sigma_{\Delta}=-2.30$. It makes sense to suggest that this star has already encountered another star in the cluster, hence the disturbed proper motion. If the system was originally a soft binary with a relatively low binding energy, it could hardly retain its genuine component upon the encounter. But if the third star was of comparable mass to the secondary component in the binary, an exchange encounter could possibly have taken place, when the total change of energy in the system is negative and larger in absolute value than the kinetic energy of the third star (Heggie 1975). The genuine secondary component could have escaped the system. Such exchanged components may account for the physical binary stars with non-coeval components, discussed in Pourbaix (1999). Provided a wide spread of ages inside a cluster, exchange encounters can generate non-coeval pairs quite efficiently. On the contrary, an encounter with a field star will normally disrupt a soft binary.

\section{Discussion}

A wide spread of ages in open clusters was proposed by Herbig (1962). His argument was based on a comparison of the turn-off age and the gravitational contraction age, as determined from most massive and least massive dwarfs in a cluster. The theory of stellar evolution predicts that coeval stars of great masses evolve from the ZAMS into the subgiant domain while the smallest mass stars still have not reached the ZAMS. Thus the blue turn-off point in the HR diagram should be accompanied by a distinct swing of the lower sequence into the pre-main-sequence domain. Herbig pointed out that in the Pleiades, which was the most suitable cluster for that analysis, such a swing was not observed where it was expected, i.e., some of the lower mass stars were found on the main sequence below the expected turn-on point.

This could be interpreted as presence of low mass stars which are considerably older than the turn-off age. It does not preclude the existence of low mass stars younger than the turn-off age, because the turn-off method is not sensitive to their presence. There might be three hardly distinguishable reasons for stars to lie above the main sequence of a cluster: 1) binarity; 2) differential reddening; 3) premain-sequence age. Herbig did not actually propose that massive stars were born later than their low mass coun- terparts, although he did suggest that a hot very massive star, once born within the molecular cloud, disperses the remaining gas in its vicinity and thus prohibits further star formation.

A kinematic segregation between strong and weak X-ray sources was recently detected in the Hyades open cluster (Makarov 2000). The internal velocity dispersion (in one component) was estimated at $0.44 \mathrm{~km} \mathrm{~s}^{-1}$ for 22 stars not detected by ROSAT, $0.32 \mathrm{~km} \mathrm{~s}^{-1}$ for 37 stars with $L_{\mathrm{X}}<1310^{28} \mathrm{erg} \mathrm{s}^{-1}$ and zero for 34 stars with $L_{\mathrm{X}}>1310^{28} \mathrm{erg} \mathrm{s}^{-1}$. Only stars with $B-V<0.7 \mathrm{mag}$ were used in order to enhance the astrometric precision over the sample. In this paper, we detect a similar effect in the Pleiades. Although both results are statistically marginally significant, together they seem to reinforce the much debated non-coeval hypothesis. According to Chandrasekhar (1942), the relaxation time of a stellar cluster is $T_{\mathrm{E}} \propto \bar{R}^{3 / 2}$, where $\bar{R}$ is approximately the core radius. This strong dependence suggests that for a given cluster, the initial time of relaxation is conditioned by the original size of the star formation site. If the progenitor cloud is small and dense, as was considered by Kroupa (1995), the relaxation time is short, and the early dynamical evolution is generally a rapid expansion of the star system and loss of numerous members. If the stars are born in a volume comparable in size to the present day cluster, the relaxation time may be tens of Myr. By Chandarsekhar's formula (5.218), assuming for the Pleiades $\bar{R}=3.5 \mathrm{pc}$ and a total mass (including the remaining gas) of $600 M_{\odot}$, $T_{E}=60 \mathrm{Myr}$, close to the age of the cluster. Thus, if the star formation took a long interval of time (>30 Myr), the last generation of stars would currently not be relaxed.

Another useful constraint may be obtained from the mean free path time $T_{\mathrm{D}}$ for the cluster, that is the time before the next encounter with another star. Using Chandrasekhar's formula (5.227), we obtain $T_{\mathrm{D}}=36 \mathrm{Myr}$ for an rms internal velocity of $0.6 \mathrm{~km} \mathrm{~s}^{-1}$ in a virialized state. This provides an age estimate for the youngest Xray stars. The oldest $\mathrm{B}$ and $\mathrm{A}$ stars in the Pleiades are probably 70 Myr old. Lada et al. (1984) in their simulations consider an option when a gravitationally bound cluster is formed with its members at rest with respect to the centre of gravity of the cloud, i.e., an initially nonvirialized cluster. This may happen if the Jeans length is much larger than a characteristic turbulent scale length in the protostellar cloud. Obviously, the new stars after a short time ( $\sim 8 \mathrm{Myr}$ for the Pleiades) will be involved in a general collapse. Thus, the non-coeval hypothesis allows to predict the following observable phenomena. The latest generation stars were born into an ambient steady dynamical system occupying a sizeable volume in space. Their young age is accompanied by a relatively high level of X-ray activity. Their original motion at birth was nearly zero with respect to the centre of mass since they had been generated non-virialized. Since their age is less than the mean free path time, they have not encountered other 
stars yet, thus retaining their unperturbed motion; many of them remain in soft binaries.

If a large part of the observed spread of $L_{\mathrm{X}}$ inside an open cluster can be ascribed to a spread of ages, the relation between these characteristics is more strict than was envisaged before. The dependence between $\log$ (Age) and $\log L_{\mathrm{X}}$, based on median luminosities in a few nearby clusters, is represented with a straight line of slope -0.97 (Patten \& Simon 1996). The observed interquartile range of $L_{\mathrm{X}}$ for the $\mathrm{G}$ stars in the Pleiades (Table 9 loc cit) implies a spread of ages roughly 15 to $150 \mathrm{Myr}$. This implies that some of the solar-type stars in the cluster are considerably older than the more massive B and A stars.

Acknowledgements. The work of one of the authors (VVM) was supported by the Danish Space Board. C. Fabricius kindly provided his software for dealing with the Tycho-2 Catalogue. S. Urban helped us to improve the text. Extensive use has been made of the Simbad data base operated at CDS, Strasbourg, France.

\section{References}

Bouvier, J., Rigaut, F., \& Nadeau, D. 1997, A\&A, 320, 74 Chandrasekhar, S. 1942, Principles of Stellar Dynamics (The University of Chicago Press, Chicago, Illinois)

ESA 1997, The Hipparcos and Tycho Catalogues, ESA SP-1200

Gatewood, G., de Jonge, J. H., \& Han, I. 2000, ApJ, 533, 938

Geffert, M., Kümmel, M. W., \& Schmidt, H. 1995, A\&AS, 112, 229

Gunn, J. E., Griffin, R. F., Griffin, R. E. M., \& Zimmerman, B. A. 1988 , AJ 96,198

Heggie, D. C. 1975, MNRAS, 173, 729

Herbig, G. H. 1962, ApJ, 135, 736

Hertzsprung, E. 1947, Ann. Leiden Obs., 19, 1A

Høg, E., Bässgen, G., Bastian, U., et al. 1997, A\&A, 323, L57

Høg, E., Fabricius, C., Makarov, V. V., et al. 2000a, A\&A, 355, L27

Høg, E., Fabricius, C., Makarov, V. V., et al. 2000b, Guide to the Tycho-2 Catalogue, on the Tycho-2 CD-ROM (Copenhagen University Observatory)
Høg, E., Fabricius, C., Makarov, V. V., et al. 2000c, A\&A, 357, 367

Jones, B. F. 1970, AJ, 75, 563

Kendall, M. G., \& Stuart, A. 1973, The Advanced Theory of Statistics (Griffin, London)

Kroupa, P. 1995, MNRAS, 277, 1522

Lada, C. J., Margulis, M., \& Dearborn, D. 1984, ApJ, 285, 141

Loktin, A. V., \& Matkin, N. V. 1994, Astron. Astrophys. Trans., 4, 153

Makarov, V. V., Odenkirchen, M., \& Urban, S. 2000, A\&A, 358,923

Makarov, V. V. 2000, A\&A, 358, L63

Mason, B. D., Hartkopf, W. I., \& McAlister, H. A. 1993, AJ, 106, 637

Mermilliod, J.-C., Rosvick, J. M., Duquennoy, A., \& Mayor, M. 1992, A\&A, 265, 513

Meyer, C., Rabbia, Y., \& Froschle, M. 1995, A\&AS, 110, 107

Micela, G., Sciortino, S., Harnden Jr., F. R., et al. 1999, A\&A, 341,751

Narayanan, V. K., \& Gould, A. 1999, ApJ, 515, 256

Patience, J., Ghez, A. M., Reid, I. N., et al. 1998, AJ, 115, 1972

Patten, B. M., \& Simon, T. 1996, ApJS, 106, 489

Perryman, M. A. C., Brown, A. G. A., Lebreton, Y., et al. 1998, A\&A, 331, 81

Pinsonneault, M. H., Stauffer, J., Soderblom, D., et al. 1998, ApJ, 504, 170

Pourbaix, D. 1999, A\&A, 348, 127

Raboud, D., Mermilliod, J.-C. 1998, A\&A, 329, 101

Robichon, N., Arenou, F., Mermilliod, J.-C., \& Turon, C. 1999, A\&A, 345, 471

Smart, W. M. 1938, Stellar Dynamics (Cambridge University Press)

Stern, R. A., Schmitt, J. H. M. M., \& Kahabka, P. T. 1995, ApJ, 448, 683

Stauffer, J. R., Caillault, J.-P., Gagné, M., et al. 1994, ApJS, 91,625

Turon, C., Crézé, M., Egret, D., et al. 1992, The Hipparcos Input Catalogue, ESA SP-1136

Urban, S. E., Corbin, T. E., \& Wycoff, G. L. 1998, AJ, 115, 2161

Urban, S. E., Wycoff, G. L., \& Makarov, V. V. 2000, AJ, 120, 501

Voges, W., Aschenbach, B., Boller, Th., et al. 1999, A\&A, 349, 389 